\title{
Rapid formation of massive black holes in close proximity to embryonic protogalaxies
}

\section{Regan, John A.}

2017-04

Regan , J A , Visbal , E , Wise , J H , Haiman , Z , Johansson , P H \& Bryan , G L 2017 , ' Rapid formation of massive black holes in close proximity to embryonic protogalaxies ' , Nature Astronomy , vol. 1 , no. 4 , 0075 . https://doi.org/10.1038/s41550-017-0075

http://hdl.handle.net/10138/307550

https://doi.org/10.1038/s41550-017-0075

other

acceptedVersion

Downloaded from Helda, University of Helsinki institutional repository.

This is an electronic reprint of the original article.

This reprint may differ from the original in pagination and typographic detail.

Please cite the original version. 


\section{LETTER}

\section{Rapid Formation of Massive Black Holes in close proximity to Embryonic Proto-Galaxies}

${ }^{*}$ John A. Regan ${ }^{1,2}$, Eli Visbal ${ }^{3,4}$, John H. Wise ${ }^{5}$, Zoltán Haiman ${ }^{3,6}$, Peter H. Johansson ${ }^{7}$ \& Greg L. Bryan ${ }^{3,4}$

${ }^{1}$ Institute for Computational Cosmology, Durham University, South Road, A Durham, DH1 3LE, UK ${ }^{2}$ Centre for Astrophysics \& Relativity, School of Mathematical Sciences, Dublin City University, Glasnevin, D09 Y5N0, Dublin, Ireland ${ }^{3}$ Department of Astronomy, Columbia University, 550 West 120th Street, New York, NY, 10027, U.S.A. ${ }^{4}$ Center for Computational Astrophysics, Flatiron Institute, 162 5th Ave, New York, NY, 10003, U.S.A. ${ }^{5}$ Center for Relativistic Astrophysics, Georgia Institute of Technology, 837 State Street, Atlanta, GA 30332, USA ${ }^{6}$ Department of Physics, New York University, New York, NY 10003 , USA ${ }^{7}$ Department of Physics, University of Helsinki, Gustaf Hällströmin katu 2a, FI-00014 Helsinki, Finland

The Direct Collapse Black Hole (DCBH) scenario provides a solution for forming the massive black holes powering bright quasars observed in the early Universe. A prerequisite for forming a DCBH is that the formation of (much less massive) Population III stars be avoided - this can be achieved by destroying $\mathrm{H}_{2}$ via Lyman-Werner $(L W)$ radiation $\left(E_{\mathrm{LW}}=\mathbf{1 2 . 6} \mathrm{eV}\right)$. We find that two conditions must be met in the proto-galaxy that will host the DCBH. First, prior star formation must be delayed; this can be achieved with a background $\mathbf{L W}$ flux of $\left.\mathbf{J}_{\mathrm{BG}} \gtrsim 100 J_{21}\right]^{\dagger}$, Second, an intense burst of $L W$ radiation from a neighbouring star-bursting proto-galaxy is required, just before the gas cloud undergoes gravitational collapse, to finally suppress star formation completely. We show here for the first time using high-resolution hydrodynamical simulations, including full radiative transfer, that this low-level background, combined with tight synchronisation and irradiation of a secondary proto-galaxy by a primary proto-galaxy, inevitably moves the secondary proto-galaxy onto the isothermal atomic cooling track, without the deleterious effects of either photo-evaporating the gas or polluting it by heavy elements. These, atomically cooled, massive proto-galaxies are expected to ultimately form a DCBH of mass $10^{4}-10^{5} M_{\odot}$.

The appearance of SMBHs at very early times in the Universe $e^{1.3}$ is a challenge to our understanding of star and black hole formation in the early Universe. Either a select few Population III (PopIII) remnants must have undergone periods of prodigious growth ${ }^{4.8}$ or SMBHs could have been formed through massive galactic collisions 9.10 or alternatively SMBH seeds must have been massive $\left(\sim 10^{4}-10^{6}\right.$ $\mathrm{M}_{\odot}$ ) to begin with 11 . We here investigate the latter, so-called, DCBH scenario. To probe the unique combination of a background radiation field in tandem with an intense proximate burst we perform a series of high resolution radiation-hydrodynamic simulations using the adaptive mesh refinement code Enzo ${ }^{12}$ together with the Grackle multi-species library for solving the primordial $(\mathrm{H}+\mathrm{He})$ chemistry network and regulating the radiation backgrounds $\sqrt{13}$. Radiative transfer is handled by Enzo's MORAY ray tracing package 14 .

We employ relatively mild backgrounds from between $\mathrm{J}_{\mathrm{BG}}=100 J_{21}$ up to $\mathrm{J}_{\mathrm{BG}}=200 J_{21}$ for an effective background $J_{21}$ is the intensity of background radiation in units of cited in the lierature where background fields of $\gg 1000 J_{21}$ are typically invoked

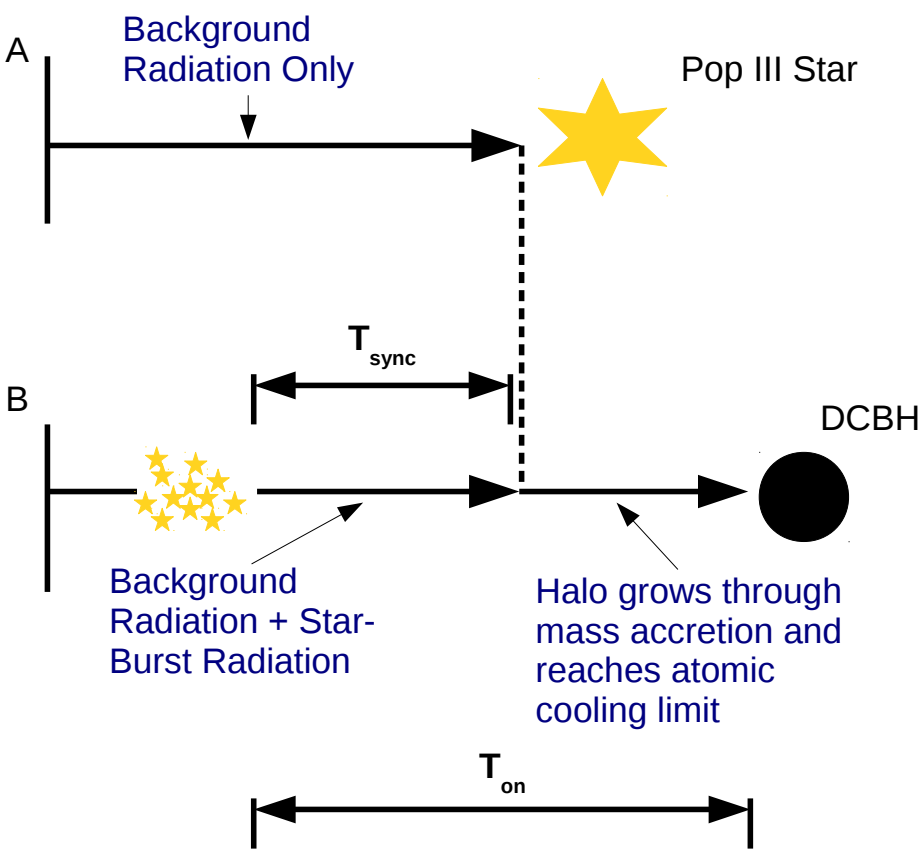

Figure 1 | Modelling Synchronised Haloes. The synchronised proto-galaxy scenario. With only a background field in operation a (delayed) Pop III star forms due to $\mathrm{H}_{2}$ cooling (Case A). If a nearby star-burst galaxy, in conjunction with the background, provides the critical LW flux required then a DCBH can form in an atomic cooling halo (Case $\mathrm{B}$ ). $\mathrm{T}_{\text {sync }}$ is defined as the time between the star-burst turning on and the point at which a PopIII would have formed. $\mathrm{T}_{\mathrm{on}}$ is the time taken for an atomic cooling halo to collapse and form a DCBH (or the minimum time the source must shine for).

temperature of $30,000 \mathrm{~K}$. This is the effective temperature expected from a population of metal-free and partially metal-enriched stars in the early Universe. This (relatively mild) global background radiation field is sufficient to delay the collapse but will not prevent the formation of $\mathrm{H}_{2}$ as the hal ${ }^{\mp}$ mass increases. To model the nearby source we use the "synchronised pairs" scenario in which a proto-galaxy (secondary halo) is exposed to the intense radiation from a star-burst (primary halo) that is sufficiently near-by ${ }^{15 \cdot 18}$ and tightly synchronised in time ${ }^{19}$. The model is illustrated in Figure 1. If the primary halo crosses the atomic cooling threshold (and begins forming stars through molecular collapse) closely in time before a neighbouring, secondary halo, the primary halo can bombard the secondary halo with a critical flux of LW radiation. This bombardment destroys the $\mathrm{H}_{2}$ in the secondary halo due to the extremely high flux of the primary halo. It provides the final push to the secondary halo forcing the halo onto the atomic cooling track leading to DCBH formation. Forming massive black holes in this way is a promising way of producing massive black hole seeds in the early Universe.

\footnotetext{
$\ddagger$ halo encompasses both the proto-galaxy and the dark matter structure surrounding the proto-galaxy
} 
Further consideration must be given to preventing both metal

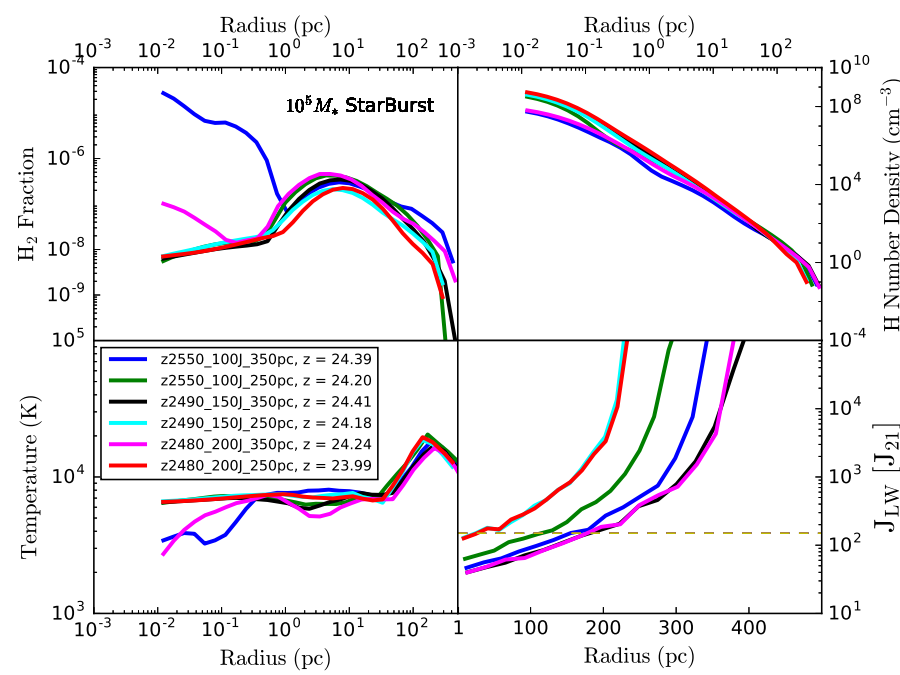

Figure 2 | Ray Profiles for six selected haloes. A selection of Ray Profiles from simulations using a star-bursting galaxy source as the local radiation source. For an atomic cooling halo to form the distance to the source must, in general, be less than approximately $300 \mathrm{pc}$. Four simulations make it to the atomic cooling track (green, black, cyan \& red lines) while two simulations (blue \& magenta) end up on the molecular cooling track. The simulations which "failed" to achieve an isothermal collapse both show marked increases in $\mathrm{H}_{2}$ towards the very centre of the halo. The golden dashed line in the bottom right panel shows the level of the uniform background radiation employed (set at $\mathrm{J}_{\mathrm{BG}}=150 J_{21}$ in this illustrative case).

contamination and photo-evaporation (from photons with $\mathrm{E}>13.6$ $\mathrm{eV}$ ) from neighbouring haloes. Metal cooling will rapidly reduce the temperature of the collapsing gas resulting in strong fragmentation (the cooling time at a density of $\mathrm{n}_{\mathrm{HI}} \sim 100 \mathrm{~cm}^{-3}$ is about $10 \mathrm{Myr}$ for gas with a metallicity of $Z=10^{-4}-10^{-3} \mathrm{Z}_{\odot}$ ). Therefore, for the Jeans mass to remain large, metal pollution from nearby galaxies must be avoided (although this can be mitigated by inefficient or slow mixing due to external metal enrichmen ${ }^{20}$ ). In addition, photo-evaporation in haloes exposed to star-burst radiation for longer than $\sim 40$ Myrs from a source with luminosity $\mathrm{L}=1.64 \times 10^{41} \mathrm{erg} / \mathrm{s}$ and a separation of between 0.5 and $1 \mathrm{kpc}$ was observed in the simulations of Regan et al. $(2016)^{21}$, effectively limiting the time available for creating a pristine atomic cooling halo $(\mathrm{ACH})$ using a nearby neighbour.

Our results show that in order to achieve an $\mathrm{ACH}$ the separation, $\mathrm{R}_{\text {sep }}$, between the primary and secondary haloes needs to be less than approximately $300 \mathrm{pc}$ for the stellar luminosity of the primary halo adopted in our model $\left(1.2 \times 10^{52}\right.$ photons/sec, or a stellar mass of $\left.\mathrm{M}_{*} \sim 10^{5} \mathrm{M}_{\odot}\right)$. This critical distance will vary as $\mathrm{d}_{\text {crit }} \propto \sqrt{\mathrm{M}_{*}}$ but for the luminosities examined in this paper will be approximately 300 pc. We define the synchronisation time as the time between when the primary halo is turned on and when the gas in the secondary halo would have collapsed in the absence of the primary halo - see Figure 1. The synchronisation times that successfully produce an ACH can range from a few kyr to a few Myr (see column 6 in Supplementary Data Table 1). Short synchronisation times reduce the probability of metal pollution and/or photo-evaporation from the primary halo.

In Figure 2 we show a selection of Ray Profiles using our primary halo as the local galactic source (the stellar mass of the primary halo was determined from high resolution simulations of the high redshift Universe ${ }^{22}$ ). For each simulation, we show the temperature, $\mathrm{H}_{2}$ fraction, hydrogen number density and the intensity in the LW band as a function of distance from the center of the secondary halo. Of the six cases examined in Figure 2 four collapse nearly isothermally and form ACHs, while the two others form excessive $\mathrm{H}_{2}$, cooling significantly.
Three of the "successful" simulations lie within the critical distance of $300 \mathrm{pc}$, with the fourth at $388 \mathrm{pc}$. The thermal profile of the fourth simulation is not as smooth as the closer-in simulations and this case is therefore likely close to a tipping point (i.e. a slightly larger separation would likely have resulted in a non-isothermal collapse). The virial radius of our secondary halo is approximately $300 \mathrm{pc}$ - meaning that for the neighbouring source to have the greatest probability of completely suppressing $\mathrm{H}_{2}$ cooling the virial radii of the primary and secondary must overlap $\frac{19}{}$. Primary sources which lie outside of the virial radius of the secondary do not, in general, provide sufficient flux without unrealistically high star-formation efficiencies.

How spatially and temporally correlated do the halo pairs need to be? In Figure 3 we show the average separation against the synchronisation

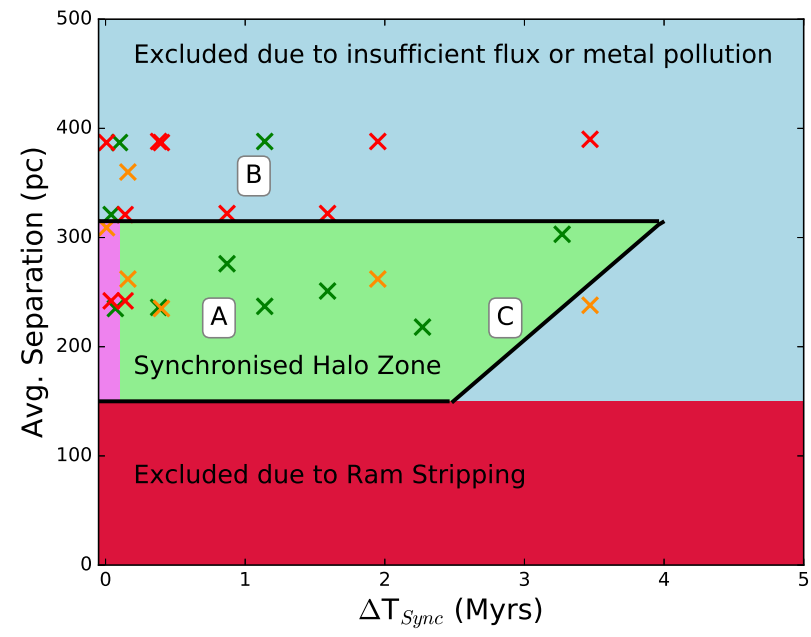

Figure 3 The Synchronised Halo Zone. This figure sums up the results of the suite of simulations conducted during this study. Plotted on the x-axis is the synchronization time, and on the $y$-axis the average separation between the source and the target halo. As the synchronization time increases, the likelihood of attaining a DCBH diminishes as the risk of metal pollution and photo-evaporation increases. Green crosses indicate cases where an atomic cooling halo was achieved, orange crosses indicate cases where an atomic cooling halo was achieved but the background was at our highest value of $\mathrm{J}_{\mathrm{BG}}=200 J_{21}$. Red crosses indicate simulations in which a molecular core formed in the centre of the collapsing halo. The green region in the centre is the region in which we find atomic haloes most likely to form. We also tentatively indicate (purple hatched region on the extreme left) a "point of no return" at which point we expect the formation of PopIII stars to be unstoppable independent of the intensity of the nearby start-burst.

time, $\mathrm{T}_{\text {sync }}$, for all of the simulations conducted in this study. As noted above, for separations of $\mathrm{R}_{\text {sep }} \gtrsim 300 \mathrm{pc}$ we tend not to form ACHs and instead a critical level of $\mathrm{H}_{2}$ builds up leading to a non-isothermal collapse. Due to the possibility of metals from supernova explosions polluting the pristine environment of the secondary halo and the detrimental effects of long term exposure of the gas to ionising radiation we disfavour sources which must be "on" for more than 10 Myrs (which is comparable to the lifetime of massive stars) before the initial collapse occurs. We therefore do not probe backgrounds with $J_{B G}<100 J_{21}$. As a result of these constraints, regions at the top and to the right in Figure 3 are excluded. Ram pressure stripping will affect haloes which get too close to one another and thus the bottom section of the graph is excluded for this reason 19:23 (see Methods section for further discussion). This leaves a (green) region in the left centre of the Figure which allows for the formation of DCBHs. The crosses are results from the suite of simulations (see Supplementary Data Table 1). The simulations which formed an atomic cooling halo are labelled with green crosses and live in this region. Orange crosses indicate simulations which formed an atomic cooling halo but for which the background was set to $\mathrm{J}_{\mathrm{BG}}=$ 
$200 J_{21}$ (the maximum level in our study). This is quite a strong background and may be beyond even the most clustered regions ${ }^{24}$. Red crosses indicate simulations in which a non-isothermal collapse was observed due to $\mathrm{H}_{2}$ cooling and in which a DCBH is therefore unlikely to form.

Three regions of particular interest are marked on the plot as A, B \& C. Region A is where most of our simulation results cluster. Short ( $_{\text {sync }} \lesssim 2 \mathrm{Myr}$ ) synchronisation times combined with close separations almost always resulted in an $\mathrm{ACH}$. Region $\mathrm{B}$ is outside our "Synchronised Halo Zone" (SHZ) and here the majority of the simulations show that the flux received by the secondary is too small and an atomic cooling halo does not form. Nonetheless four atomic cooling haloes appear outside the SHZ. Two of the green crosses are from runs with a background of $\mathrm{J}_{\mathrm{BG}}=150 J_{21}$ while one is from a run with a background of $\mathbf{J}_{\mathrm{BG}}=100 J_{21}$. The orange cross had a background of $\mathrm{J}_{\mathrm{BG}}=200 J_{21}$. Similarly, two molecular collapses are seen inside the SHZ showing that fluctuations limit the accuracy of the SHZ boundary at approximately the $10 \%$ level. Region C indicates the tip of the SHZ. As the synchonisation time gets longer the risk of photoionisation and/or metal pollution becomes larger. Synchronisation limits will vary depending on the exact environmental conditions, the luminosity, the spectrum and the distance to the primary halo, we therefore only probed the limits of the separation and the limits of the synchronisation (i.e. how short/extended can the synchronisation time be).

Forming seeds for the SMBHs we observe at the centres of the most massive galaxies and as very high redshift quasars is an outstanding problem in modern astrophysics. We show here that the combination of a relatively mild "local" radiation background field due to the clustering of early galaxies plus a nearby star-burst event is the perfect trigger for the creation of such an atomic cooling halo. The local background serves to delay PopIII star formation allowing a sufficiently massive halo to develop. A nearby (synchronised) star-burst can then irradiate the now massive halo with a flux greater than the critical LW flux pushing the collapsing halo onto the atomic cooling track while avoiding the deleterious effects of photo-evaporation or metal pollution. We find that a "synchronised halo zone" exists where the separation between the neighbouring haloes is between approximately $200 \mathrm{pc}$ and $300 \mathrm{pc}$ and the synchronisation time between the halo's evolution is less than approximately 4 Myrs ("on" time $<10$ Myrs). Furthermore, we find the mass inflow rates onto the central object are greater than $0.1 \mathrm{M}_{\odot} \mathrm{yr}^{-1}$ over several decades in radius.

Close halo pairs with tight synchronisation times should easily fulfill the number density requirements of SMBHs at high redshif ${ }^{[15 ; \mid 19: 24}$ (see methods section for further details and a calculation of expected number densities) and may play a significant role in the formation of all SMBHs. Upcoming observations with the JWST will search for DCBH candidates up to redshifts of $z \sim 25$, and may be able to identify them based on their unique spectral signatures 2526 . A detection of a DCBH candidate together with star-forming galaxies in close proximity would validate the synchronisation mechanism.

1. Fan, X., Carilli, C. L. \& Keating, B. Observational Constraints on Cosmic Reionization. Ann. Rev. Astron. Astrophys. 44, 415-462 (2006). arXiv: astro-ph/0602375

2. Mortlock, D. J. et al. A luminous quasar at a redshift of $z=7.085$. Nature 474, 616-619 (2011). 1106.6088

3. Wu, X.-B. et al. An ultraluminous quasar with a twelve-billion-solar-mass black hole at redshift 6.30. Nature 518,512-515 (2015). 1502.07418

4. Madau, P. \& Rees, M. J. Massive Black Holes as Population III Remnants. Astrophys. J. Lett. 551, L27-L30 (2001). arXiv: astro-ph/0101223

5. Tanaka, T. \& Haiman, Z. The Assembly of Supermassive Black Holes a High Redshifts. ArXiv e-prints (2008). 0807.4702

6. Madau, P., Haardt, F. \& Dotti, M. Super-critical Growth of Massive Black Holes from Stellar-mass Seeds. Astrophys. J. Lett. 784, L38 (2014). 1402.6995

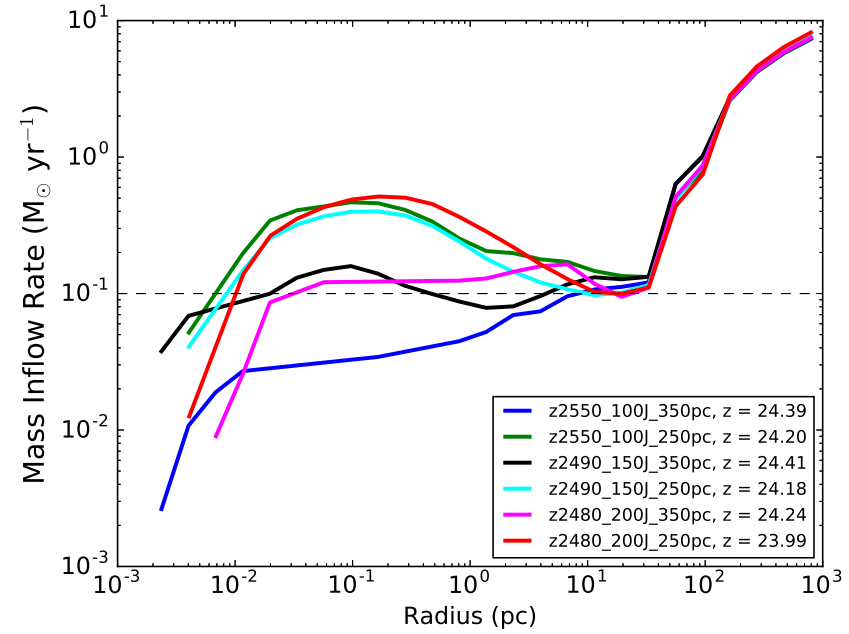

Figure 4 | Mass Inflow Rates. Mass Inflow Rates into the centre of collapsing halo for the same subset of simulations as shown in Figure 2. The mass inflow rates are calculated by determining the flux in spherical shells surrounding the central object. Focusing on the core of the collapsing halo where $R<10 \mathrm{pc}$ the mass inflow rates are typically much greater than $0.1 \mathrm{M}_{\odot} \mathrm{yr}^{-1}$ with values peaking at around $0.75 \mathrm{M}_{\odot} \mathrm{yr}^{-1}$ at $\mathrm{R} \sim 5 \times 10^{-2} \mathrm{pc}$. Interestingly, the highest mass inflow rates are seen for the simulations in which the flux is largest (i.e. the smallest separations). Finally, accretion rates greater than $0.1 \mathrm{M}_{\odot} \mathrm{yr}^{-1}$ are sustained over several decades in radius. Below a scale of $\mathrm{R} \approx 3 \times 10^{-2} \mathrm{pc}$ the inflow rates fall due to limited resolution effects below this scale.

7. Lupi, A. et al. Growing massive black holes through supercritical accretion of stellar-mass seeds. Mon. Not. R. Astron. Soc. 456, 2993-3003 (2016). 1512.02651

8. Inayoshı, K., Haiman, Z. \& Ostriker, J. P. Hyper-Eddington accretion flows on to massive black holes. Mon. Not. R. Astron. Soc. 459, 3738-3755 (2016). 1511.02116

9. Mayer, L., Kazantzidis, S., Escala, A. \& Callegari, S. Direct formation of supermassive black holes via multi-scale gas inflows in galaxy mergers. Nature 466, 1082-1084 (2010). 0912.4262

10. Mayer, L. et al. Direct Formation ot Supermassive Black Holes in Metalenriched Gas at the Heart of High-redshift Galaxy Mergers. Astrophys. J. 810, 51 (2015). 1411.5683

11. Loeb, A. \& Rasio, F. A. Collapse of primordial gas clouds and the formation of quasar black holes. Astrophys. J. 432, 52-61 (1994). arXiv:astro-ph/9401026

12. Bryan, G. L. et al. ENZO: An Adaptive Mesh Refinement Code for Astrophysics. Astrophys. J. Suppl. 211, 19 (2014). 1307.2265

13. Smith, B. D. et al. Grackle: a Chemistry and Coolıng Library for Astrophysics. ArXiv e-prints:1610.09591 (2016). 1610.09591

14. Wise, J. H. \& Abel, T. ENZO+MORAY: radiation hydrodynamics adaptive mesh refinement simulations with adaptive ray tracing. Mon. Not. $R$ Astron. Soc. 414, 3458-3491 (2011). 1012.2865

15. Dijkstra, M., Haiman, Z., Mesinger, A. \& Wyithe, J. S. B. Fluctuations in the high-redshift Lyman-Werner background: close halo pairs as the origin of supermassive black holes. Mon. Not. R. Astron. Soc. 391, 19611972 (2008). 0810.0014

16. Yue, B., Ferrara, A., Salvaterra, R., Xu, Y. \& Chen, X. The brief era of direct collapse black hole formation. Mon. Not. R. Astron. Soc. 440, 1263-1273 (2014). 1402.5675

17. Agarwal, B., Davis, A. J., Khochfar, S., Natarajan, P. \& Dunlop, J. S. Unravelling obese black holes in the first galaxies. Mon. Not. R. Astron. Soc. 432, 3438-3444 (2013). 1302.6996

18. Agarwal, B., Dalla Vecchia, C., Johnson, J. L., Khochfar, S. \& Paardekooper, J.-P. The First Billion Years project: birthplaces of direct collapse black holes. Mon. Not. R. Astron. Soc. 443, 648-657 (2014). 1403.5267

19. Visbal, E., Haiman, Z. \& Bryan, G. L. Direct collapse black hole formation from synchronized pairs of atomic cooling haloes. Mon. Not. R. Astron. Soc. 445, 1056-1063 (2014). 1406.7020

20. Smith, B. D., Wise, J. H., O'Shea, B. W., Norman, M. L. \& Khochfar, S. The first Population II stars formed in externally enriched mini-haloes. Mon. Not. R. Astron. Soc. 452, 2822-2836 (2015). 1504.07639

21. Regan, J. A., Johansson, P. H. \& Wise, J. H. Forming Super-Massive Black Hole Seeds under the Influence of a Nearby Anisotropic MultiFrequency Source. Mon. Not. R. Astron. Soc. (2016). 1511.00696 
22. Xu, H., Wise, J. H. \& Norman, M. L. Population III Stars and Remnants in High-redshift Galaxies. Astrophys. J. 773, 83 (2013). 1305.1325

23. Chon, S., Hirano, S., Hosokawa, T. \& Yoshida, N. Cosmological Simulations of Early Blackhole Formation: Halo Mergers, Tidal Disruption, and the Conditions for Direct Collapse. ArXiv e-prints (2016). 1603.08923

24. Ahn, K., Shapiro, P. R., Iliev, I. T., Mellema, G. \& Pen, U.-L. Ihe Inhomogeneous Background $\mathrm{Of}_{2}$-Dissociating Radiation During Cosmic Reionization. Astrophys. J. 695, 1430-1445 (2009). 0807.2254

25. Pacucci, F., Ferrara, A., Volonteri, M. \& Dubus, G. Shinıng in the dark: the spectral evolution of the first black holes. Mon. Not. R. Astron. Soc. 454, 3771-3777 (2015). 1506.05299

26. Natarajan, P. et al. Unvelling the tirst black holes with JWST: multiwavelength spectral predictions. ArXiv e-prints:1610.05312 (2016) 1610.05312

\section{METHODS}

\section{Simulation Setup.}

We use the adaptive mesh refinement code Enzo in this study. Enzo uses an N-body adaptive particle-mesh solver to follow the dark matter dynamics. It solves the hydrodynamics equations using the second-order accurate piecewise parabolic method, while an HLLC Riemann solver ensures accurate shock capturing with minimal viscosity. Rather that using the internal chemistry solver we use a modified version of the Grackle chemistry library which has been updated with the latest rates for modelling collapse in the face of radiation background $\$ 27,28$. The chemical network includes 33 separate chemical reactions (see Table 1 from 21 ) from 10 species - $\mathrm{H}, \mathrm{H}^{+}, \mathrm{He}, \mathrm{He}^{+}, \mathrm{He}^{++}, \mathrm{e}^{-}, \mathrm{H}_{2}, \mathrm{H}_{2}^{+}, \mathrm{H}^{-}$and $\mathrm{HeH}^{+}$.

The initial conditions are taken from Regan et al. (2015) ${ }^{29}$ and were generated with the MUSIC initial conditions generato ${ }^{30}$. Haloes were initially located by running a large suite of dark matter only simulations in a cosmological context. Realisations were then selected by choosing simulations in which massive dark matter haloes formed relatively early and thus represented high "sigma" peaks in the primordial density field Simulations were then rerun with gas dynamics included. All simulations were run within a box of size $2 h^{-1} \mathrm{Mpc}$ (comoving), the root grid size was $256^{3}$ and we employed three levels of nested grids. Within the most refined nested grid the dark matter resolution was $\mathrm{M}_{\mathrm{DM}} \sim 103 \mathrm{M}_{\odot}$. In order to increase further the dark matter resolution we split the dark matter particles 31 at a redshift of $\mathrm{z}=40$, well before the onset of the collapse. This has no adverse effect on the dynamics of the collapse but is a necessary step in high resolution simulations 29 . The baryon resolution is set by the size of the highest resolution cells within the grid. Grids are refined in Enzo whenever, user defined, criteria are breached. We allow refinement of grid cells based on three physical measurements: (1) the dark matter particle overdensity, (2) the baryon overdensity and (3) the Jeans length. The first two criteria introduce additional meshes when the overdensity $\left(\frac{\delta \rho}{\rho_{\text {mean }}}\right)$ of a grid cell with respect to the mean density exceeds 8.0 for baryons and/or dark matter. Furthermore, we set the MinimumMassForRefinementExponent parameter to -0.1 making the simulation super-Lagrangian and therefore reducing the threshold for refinement as higher densities are reached. For the final criteria, we set the number of cells per Jeans length to be 16 in these simulations. We set the maximum allowed refinement level to 18 . This means that we reach a maximum grid resolution of $\Delta x \sim 2 \times 10^{-3} \mathrm{pc}$ (physical at $\mathrm{z}=25$ ).

\section{Choosing a background radiation field.}

The vast majority of studies undertaken to investigate the destruction of $\mathrm{H}_{2}$ by an external radiation field have used a uniform background radiation field to demonstrate the viability of the mechanism. The general consensus has been that a critical LW intensity of $\mathrm{J} \gtrsim 1000 J_{21}$ is needed ${ }^{32.35}$ for a blackbody background with an effective temperature of $10^{5} \mathrm{~K}$ However, achieving a background of $\mathrm{J}_{\mathrm{BG}} \gtrsim 1000 J_{21}$ is very unlikely at the very high redshifts of interest $(z \gtrsim 2 5 \longdiv { 2 4 }$. Instead what is much more likely to create the required flux is a mild background $\left(\mathrm{J}_{\mathrm{BG}} \sim 100-200 J_{21}\right)$ augmented by a local burst of LW radiation from one or more nearby

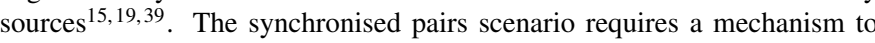

\footnotetext{
$¥$ A background intensity approximately an order of magnitude lower would be sufficien for low-mass stars with an effective temperature of $T=10^{4} K^{32,36}$, however the stellar mass required is then significantly higher 37,38 .
}

delay the formation of PopIII stars so that neighbouring haloes can build sufficient mass to cross the atomic cooling threshold in near synchronisation. In this study we impose an external background field to facilitate this delay 40,41 . For the halo studied in this work we found, in agreement with other studies in the literature, that a background radiation field of $\sim$ $1000 J_{21}$ is required to form an atomic halo with a blackbody effective temperature of $30000 \mathrm{~K}$ (when only a global background is considered). In Extended Data Figure 1 we have plotted the temperature as a function of radius for six different background fields. This figure contains no local source and shows the impact of the background radiation field only. The "Ctrl" field has no background radiation field invoked and so the halo collapses earliest in this case. As the strength of the radiation field is increased the collapse is delayed to increasingly longer times until eventually the collapse becomes isothermal and the halo collapses under the influence of atomic hydrogen cooling only.

We refer to these background fields as "local backgrounds". These are intensities produced by a collection of nearby galaxies which produce radiation which locally acts like a uniform background. The local background delays PopIII star formation in the secondary halo of our simulations. This is crucially important in the context of exploring the temporal elements of our model. If the local background is too weak then the conditions for halo synchronisation cannot be met (although streaming motions and/or rapid collapse, which are not included in our simulations, could obviate the need for the background; see below). In principle, the star-burst radiation could compensate for the lower background field. This requires, however, the star-burst to shine for a longer time, increasing the risk of metal pollution or photo-evaporation. In this study we examine background radiation fields, $\mathrm{J}_{\mathrm{BG}}$, with values of $100 J_{21}, 150 J_{21}$ and $200 J_{21}$. In all cases we initiate our background radiation field at $\mathrm{z}=35$.

\section{Radiation Source.}

In order to study the effect a local radiation source (i.e. the primary halo) can have on the "target" halo (i.e. the secondary halo) we employ a massless radiation particle for which we can vary the emission intensity and source separation. The radiation particle acts as a source of radiation, the radiation is propagated by Enzo's radiative transfer scheme ${ }^{144}$ which traces individual photon packets through the AMR mesh by utilising 64 bit HEALPix algorithms 42,43 . The ray tracing scheme allows us to follow photons in the infrared, LW and hydrogen ionisation range. The exact energy levels and relative strength of each energy bin used are given in Supplementary Data Table 2. By utilising the ray tracing scheme we are able to accurately track both the column density along each ray and the level of photodissociation of different ions along the ray path. The shielding effects of different species are accounted for by the exact knowledge of the column density (and therefore the optical depth). Self-shielding of $\mathrm{H}_{2}$ is accounted for using the prescription given in Wolcott-Green et al. $(2011 \sqrt{44}$.

We control the physical distance between the primary and the secondary haloes by first running a test simulation where the radiation particle is placed at a distance of approximately $200 \mathrm{pc}$ from the source. The simulation is then run and we calculate the position of the point of maximum density averaged over multiple outputs. By doing this we know in advance how the centre of mass (COM) of the system will change as the simulation proceeds (as the initial source position is changed the COM will change but our numerical experiments showed that the change was only at the $10 \%$ level). We then choose a vector with the COM as the origin. By then placing the radiation particle at set distances along this given vector (we use the angular momentum vector for convenience) we find points where the distance to the secondary halo remains approximately constant - although some variation is expected. This mimics a scenario where the secondary halo and primary halo orbit one another.

The radiation spectrum emitted by the primary halo is modelled to be a partially metal enriched galaxy. We use a luminosity of $\sim 1.2 \times 10^{52}$ photons per second (above the $\mathrm{H}^{-}$photo-detachment energy of $0.76 \mathrm{eV}$ see Figure 2 in Regan et al. 2016a). We assume a stellar mass of $M_{*}=10^{5}$ $\mathrm{M}_{\odot}$ at $z=20$, consistent with the largest galaxies prior to reionisation in the Renaissance Simulations $s^{45}$. We then calculate its spectrum using the Bruzual \& Charlot (2003) model $\sqrt{46}$ with a metallicity of $10^{-2} \mathrm{Z}_{\odot}$ and compute the photon luminosity from it. The spectrum does not include 
emission from the nebular component and is solely due to stellar emission This spectrum is virtually identical to a PopIII source with a cluster of $9 \mathrm{M}_{\odot}$ stars 47 . However, a cluster of $10^{4}$ PopIII stars is a challenging structure to produce even under extreme condition $\sqrt{45}$ and hence we opt for the metal-enriched population. The details of the spectrum (and a comparable PopIII spectrum for comparison) is detailed in Supplementary Data Table 2.

\section{Measuring the Radiation Intensity for a Local Source.}

$\mathbf{J}_{21}$ is the standard unit used to measure radiation (background) intensities. To calculate and quote an equivalent angle-averaged intensity from a single nearby source, $J_{\mathrm{L}}$, in units of $\mathrm{J}_{21}$ we sum the contributions from radiation in the LW band only. It should be noted however that the radiation intensity includes effects from radiation at other energies also.

$$
\begin{aligned}
J^{\prime} & \equiv \frac{k_{\mathrm{H} 2 \mathrm{I}} E_{\mathrm{LW}}}{4 \pi \sigma_{\mathrm{H} 2 \mathrm{I}}\left(E_{\mathrm{LW}}\right)} \\
J_{\mathrm{L}} & \equiv \frac{J^{\prime}}{\nu_{\mathrm{H}} J_{21}}
\end{aligned}
$$

where $J^{\prime}$ is the intensity in the LW band. Here $k_{\mathrm{H} 2 \mathrm{I}}$ is the number of photo-dissociations per second for $\mathrm{H}_{2}, \sigma_{\mathrm{H} 2 \mathrm{I}}(E)$ is the average cross section for dissociation in the $\mathrm{LW}$ band $\frac{48}{4}$ and $\mathrm{E}_{\mathrm{LW}}$ is the photon energy in the LW band. Finally, $\nu_{\mathrm{H}}$ is the frequency at the hydrogen ionisation edge. $J_{\mathrm{L}}$ is now the intensity of the radiation from the local source in units of $\mathrm{J}_{21}$ normalised at the Hydrogen ionisation edge. $J_{\mathrm{L}}$ will be a function of distance from the source i.e. $J_{\mathrm{L}}=J_{\mathrm{L}}(d)$.

\section{Ram Pressure Stripping \& Tidal Disruption.}

The small separation of the primary and secondary haloes implies that they are sub-haloes of a larger parent halo. As they move through the "intracluster medium" of the parent halo, they are subject to ram pressure stripping. The ram pressure due to this movement is:

$$
P_{\mathrm{ram}}=\frac{1}{2} \rho_{\mathrm{m}} v^{2}
$$

To ensure that this ram pressure does not unbind the core of the secondary galaxy, $\mathrm{P}_{\text {ram }}$ must obey $49: 51$

$$
P_{\text {ram }} \leq \frac{\alpha G M_{\text {tot }}\left(R_{\text {core }}\right) \rho_{\text {gas }}\left(R_{\text {core }}\right)}{R_{\text {core }}},
$$

where $\rho_{\mathrm{m}}$ is the gas density the core passes through, $v$ is the relative orbital velocity of the proto-galaxy, $\alpha$ is a variable of order unity which depends on the specifics of the dark matter and gas profiles, $M_{\text {tot }}\left(R_{\text {core }}\right)$ and $\rho_{\text {gas }}\left(R_{\text {core }}\right)$ are the total mass and gas density within $R_{\text {core }}$ respectively. For the secondary halo studied here we define the core of the halo as the radius at which the gas mass dominates over the dark matter mass giving us a value of $R_{\text {core }} \sim 10 \mathrm{pc}$. Using the virial mass of the secondary halo we assume a relative orbital velocity of $20 \mathrm{~km} / \mathrm{s}$, we then use the gas density at an orbital distance of $100 \mathrm{pc}\left(\rho_{\mathrm{m}}(\mathrm{R}=100 \mathrm{pc}) \approx 1 \times 10^{-23} \mathrm{~g} \mathrm{~cm}^{-3}\right)$ from the centre of the secondary halo to get

$$
P_{\text {ram }} \approx 2 \times 10^{-8} \mathrm{~g} \mathrm{~cm}^{-1} \mathrm{~s}^{-2}
$$

while the right hand side of equation 4 gives a value of

$$
\frac{\alpha G M_{\text {tot }}\left(R_{\text {core }}\right) \rho_{\text {gas }}\left(R_{\text {core }}\right)}{R_{\text {core }}} \approx 2 \times 10^{-8} \mathrm{~g} \mathrm{~cm}^{-1} \mathrm{~s}^{-2},
$$

at a radius of $10 \mathrm{pc}$. Inside of this radius the binding energy of the halo increases approximately as $\mathrm{R}^{-2}$ and so within $10 \mathrm{pc}$ ram pressure stripping will be unable to unbind the gas. To be conservative we always constrain the radiation particle to be at a distance greater than $150 \mathrm{pc}$ from the secondary halo centre. However, while the core will remain intact some mass loss from the outer parts of the protogalaxy is inevitable. Given the secondary and primary haloes are expected to eventually merge the total mass available for the accreting DCBH is unaffected.

\section{Metal Pollution, Photo-evaporation and the Maximum Irradiation} Time.

Metal pollution, photo-evaporation or the natural end of the star-burst in the primary galaxy will ultimately limit the prospects for achieving a direct collapse black hole. A PopIII source model with individual stellar masses of $\mathrm{M}_{*} \sim 9 \mathrm{M}_{\odot}$ will have an expected lifetime of $\mathrm{T}_{*} \sim 20 \mathrm{Myrs}$ 47. After this time the stars would explode as supernovae and presumably pollute the secondary halo within a few Myrs 39 . Our galaxy model includes lower mass stars with longer lifetimes but with a more distributed IMF and so also stars with masses in excess of $9 \mathrm{M}_{\odot}$. To be conservative we assume that in cases where the primary halo must be "on" for greater than 10 Myrs that a DCBH does not form. We note that we see no evidence of photo-evaporation of the secondary halo even when the secondary is irradiated for $22 \mathrm{Myr}$ (the longest "on" time we encountered during our numerical experimentation) but since we do not include the impact of metal production and pollution we limit the maximum irradiation time to 10 Myrs.

A related issue concerning metal pollution is the impact that the background galaxies, which currently are supposed to provide the background radiation field, may have. Dijkstra et al. (2014) $\sqrt{39}$ show that the metal pollution radius scales as $\mathrm{r}_{\mathrm{s}}(\mathrm{M}, \mathrm{t}) \propto \mathrm{M}_{*}^{1 / 5} \mathrm{t}^{2 / 5}$. If we assume that the radiation field is created by a collection of 5 galaxies of similar mass to our primary galaxy then the metallicity field spreads as $\mathrm{r}_{\mathrm{kpc}, \mathrm{s}}=3 \times 10^{-2}\left(5 \times 10^{5} \mathrm{M}_{\odot}\right)^{1 / 5} \mathrm{n}^{-1 / 5} \mathrm{t}_{6}^{2 / 5}$ (Dijkstra et al. (2014) equation 5) this leads to a spread of just over $2 \mathrm{kpc}$ after 30 Myrs. This suggests that for background sources separated by a distance greater than 2 $\mathrm{kpc}$ from the primary and secondary haloes that metal pollution may not be such a large concern at the redshifts explored here.

A recent study by Chon et al. (2016) $\sqrt{23}$ examined the formation of DCBHs in a large cosmological simulation. The focus of their simulations was to determine the impact of tidal disruption and ram pressure on the formation of DCBHs. In a study of 42 DC candidates they found that two candidates successfully resulted in the rapid formation of stars within an $\mathrm{ACH}$. One of the two successful candidates was due to a scenario similar to the synchronised scenario investigated here. However, their simulations were unable to accurately track the radiation coming from the primary halo and were not focused on this scenario. In their case they found that the primary halo exceeded the atomic cooling threshold 20 - 30 Myrs before the secondary halo with the $\mathrm{LW}$ intensity exceeding the critical value. This suggests that we are quite conservative with our $10 \mathrm{Myr}$ limit on the primary halo "on" time. However, similar to this study, Chon et al. do not include the effects of metal pollution which could limit the time for which the star-burst can be active before the secondary must collapse under atomic cooling. We also note that Chon et al. did not include the impact of photoionisation in their hydrodynamics simulations, whereas we do so here, and are therefore able to show that photo-evaporation is avoided.

\section{Mass In-Flow Rates.}

Large mass inflow rates are one of the pre-requisites for forming a supermassive star (SMS). For the case of (super) massive stars the gravitational contraction timescale is much shorter than the Kelvin-Helmholtz timescale meaning that the star begins nuclear burning before it has finished accreting and in fact will continue to accrete (subject to the correct environmental conditions) throughout its lifetime. Its final mass then becomes the important quantity.

In Figure 4 we have plotted the mass inflow rates as found for the same subset of simulations shown in Figure 2. The mass inflow rates are calculated in spherical shells around the central density according to the equation

$$
\dot{M}(t)=4 \pi R^{2} \rho(R) V(R)
$$

where $\dot{M}(t)$ is the mass inflow rate, $\mathrm{R}$ is the radius, $\rho$ is the density and $\mathrm{V}(\mathrm{R})$ is the radial velocity at $\mathrm{R}$. We find that the mass inflow rates peak at approximately $0.75 \mathrm{M}_{\odot} \mathrm{yr}^{-1}$ at $\mathrm{R} \sim 5 \times 10^{-2} \mathrm{pc}$. Most importantly, mass inflow rates greater than $0.1 \mathrm{M}_{\odot} \mathrm{yr}^{-1}$ are sustained over several decades in radius. Furthermore, we find that the more intense the flux (i.e. the closer the separation) the higher the mass inflow rates. This feature is likely due to the higher LW flux experienced at this radius, which should in turn lead to high gas temperatures due to the higher dissociation rates of $\mathrm{H}_{2}$. 
The classical assumption regarding the formation of SMSs is that accretion rates greater than at $0.03 \mathrm{M}_{\odot} \mathrm{yr}^{-1}$ are required 5253. At this mass accretion rate the protostellar evolution changes completely. The stellar envelope swells greatly in radius reaching up to $100 \mathrm{AU}$. The effective stellar temperature drops to close to $5000 \mathrm{~K}$ meaning that radiative feedback from the protostar becomes ineffective at preventing continued accretion In a somewhat complementary scenario a "quasi-star" may be born when high accretion rates $\left(\dot{M}(t) \gg 0.14 \mathrm{M}_{\odot} \mathrm{yr}^{-1} \sqrt{54}\right.$ onto an already existing SMS star results in the collapse of the hydrogen core into a stellar mass sized black hole. The highly optically thick gas which keeps falling onto the black hole bringing with it angular momentum results in an accretion disk forming around the central black hole. The energy feedback inflates the innermost part of the inflow resulting in a "quasi-star". The mass loss rates from these two types of objects is an area of active research with possible negative conquences in the case of quasi-star $\sqrt{55}$ which may be absent in the case of more "normal" SMS 5 . Either way the accretion rates observed in our simulations satisfy the basic requirements that accretion rates of $\gtrsim 0.1$ $\mathrm{M}_{\odot} \mathrm{yr}^{-1}$ are found over several decades in radius.

It should be noted than below a radius of approximately $3 \times 10^{-2} \mathrm{pc}$ the inflow rates shown in Figure 4 fall. This is a purely numerical effect created by both a diminising resolution below that scale (the Jeans mass of the gas at this temperature and density is approximately $0.1 \mathrm{pc}$ ) and the fact that we stop the calculation once the gas reaches our maximum refinement level. We are therefore not evolving the gas at this scale over any significant fraction of its dynamical time. Further exploration of the inflow rate at this scale would require the adoption of sink particles.

Finally, it is not certain that if a monolithic inflow of $>0.1 \mathrm{M}_{\odot} \mathrm{yr}^{-1}$ is attained and then sustained over an extended period of up to $1 \mathrm{Myr}$ (possibly up to 10 Myrs as required for quasi stars) that a single central object will form. Fragmentation may in this case still occur within the collapsing object ${ }^{57]}$ or else within a self gravitating disk around the central objec ${ }^{58}$. However, in either case the fragments are likely to form a dense cluster and ultimately a $\mathrm{DCBH} 59$.

\section{Expected and Required LW Background.}

Our simulations indicate that a background LW intensity of $J_{\mathrm{BG}} \gtrsim 100 J_{21}$ is required for DCBH formation in the synchronised haloes scenario. Determining the precise abundance of DCBHs formed as a result of this requirement is beyond the scope of the present work, however, we argue here that such a high flux can plausibly be achieved and can potentially result in a DCBH number density large enough to explain observations of SMBHs at $z \gtrsim 6$.

The intensity of the LW background at $z \gtrsim 10$ is highly uncertain. Some theoretical estimates have found mean values between $(0.1-1) J_{21}$ at $z=25$ and roughly an order of magnitude higher values by $z=1060.62$ Even though the $z=25$ values are 2-3 orders of magnitudes below the requirement found in our simulations, such a high background may still be possible in overdense environments. The simulations of Ahn et al. $(2009)^{24}$ show that even in a relatively small box $\left(35 \mathrm{Mpc} h^{-1}\right)$ there are regions with LW flux several orders of magnitude higher than the minimum value (see their Figure 11). Given the extreme rarity of $z \sim 6$ SMBHs, with $\mathrm{M}_{\mathrm{BH}}>10^{9} \mathrm{M}_{\odot},\left(\sim 10^{-9} \mathrm{Mpc}^{-3}\right)$, these objects are likely to have formed in highly biased regions with many more dark matter haloes than the mean number density. Thus, it seems plausible that nearby clustered sources could provide the necessary flux. This could be accomplished by, for example, $\sim 5$ sources similar to our primary halo within $2 \mathrm{kpc}$ or one source 5 times brighter.

We also note that a radiation background is not the only mechanism which could potentially delay PopIII star formation. Baryon-dark matter streaming velocities ${ }^{63}$ can significantly inhibit the collapse of gas in minihaloes at very high redshif ${ }^{64 \cdot 66}$ and could reduce the required intensity of the background field by $2-3$ orders of magnitude ${ }^{19 ; .67]}$. In particular Tanaka $\& \mathrm{Li}(2014)^{\sqrt[67]{6}}$ have shown that streaming velocities can suppress molecular cooling all the way up to the ACH limit. Likewise, haloes assembling unusually rapidly via successive mergers on time-scales shorter than the $\mathrm{H}_{2}$ cooling timescale can avoid star-formation at the minihalo stage 6 We do not include all of these effects in this study, but note that they reduce the need for a strong background. Nonetheless an additional intense nearby source is always required to prevent $\mathrm{H}_{2}$ forming in the centre of the collapsing hald 69

We also point out that even if the abundance of DCBHs formed in the synchronised pair channel at $z \sim 25$ is very low, the number density at somewhat lower redshift could potentially explain the abundance of high-redshift super-massive black holes. Assuming separations similar to those in our simulations, Visbal et al. (2014) dance of synchronised pair DCBHs formed between $z=10-11$ to be $0.0003 \mathrm{Mpc}^{-3}$. If the probability distribution function of the $\mathrm{LW}$ background for these pairs matched that from Ahn et al. (2009) 24 (see their Figure 11), combining this with the $J_{\mathrm{BG}} \gtrsim 100 J_{21}$ background requirement would not reduce the number density below $\sim 10^{-9} \mathrm{Mpc}^{-3}$ (which is similar to the observed number density of high-redshift quasars). It should be noted that this rough estimate is only a lower limit due to the limited box sizes used in both calculations cited above and the fact that other large scale effects, as discussed, are neglected.

\section{Data Availability}

The numerical experiments presented in this work were run with a fork of the enzo code available from https://bitbucket.org/john_regan/enzo-3.0$\mathrm{rp}$, in particular change set d11330f. This altered version of enzo also requires an altered version of the grackle cooling library, available from https://bitbucket.org/john_regan/grackle-cversion, particularly change set d8df240.

27. Glover, S. C. O. Simulating the formation of massive seed black holes in the early Universe - I. An improved chemical model. Mon. Not. R. Astron. Soc. 451, 2082-2096 (2015). 1501.05960

28. Glover, S. C. O. Simulating the formation of massive seed black holes in the early Universe - II. Impact of rate coefficient uncertainties. Mon. Not. R. Astron. Soc. 453, 2901-2918 (2015). 1504.00514

29. Regan, J. A., Johansson, P. H. \& Wise, J. H. The effect of dark matter resolution on the collapse of baryons in high-redshift numerical simulations. Mon. Not. R. Astron. Soc. 449, 3766-3779 (2015). 1501.05650

30. Hahn, O. \& Abel, T. Multi-scale initial conditions for cosmological simulations. Mon. Not. R. Astron. Soc. 415, 2101-2121 (2011). 1103.6031

31. Kitsionas, S. \& Whitworth, A. P. Smoothed Particle Hydrodynamics with particle splitting, applied to self-gravitating collapse. Mon. Not. R. Astron. Soc. 330, 129-136 (2002). astro-ph/ 0203057

32. Shang, C., Bryan, G. L. \& Haiman, Z. Supermassive black hole formation by direct collapse: keeping protogalactic gas $\mathrm{H}_{2}$ free in dark matter haloes with virial temperatures $\mathrm{T}_{v i r} \gtrsim 10^{4} \mathrm{~K}$. Mon. Not. R. Astron. Soc. 402, 1249-1262 (2010). 0906.4773

33. Latif, M. A. et al. A UV flux constraint on the formation of direct collapse black holes. Mon. Not. R. Astron. Soc. 443, 1979-1987 (2014). 1404. 5773

34. Latif, M. A., Schleicher, D. R. G., Bovino, S., Grassi, T. \& Spaans, M. The Formation of Massive Primordial Stars in the Presence of Moderate UV Backgrounds. Astrophys. J. 792, 78 (2014). 1406.1465

35. Agarwal, B. \& Khochfar, S. Revised rate coefficients for $\mathrm{H}_{2}$ and $\mathrm{H}^{-}$ destruction by realistic stellar spectra. Mon. Not. R. Astron. Soc. 446, 160-168 (2015). 1407.4115

36. Omukai, K. Primordial Star Formation under Far-Ultraviolet Radiation. Astrophys. J. 546, 635-651 (2001). astro-ph/0011446

37. Wolcott-Green, J. \& Haiman, Z. Feedback from the infrared background in the early Universe. Mon. Not. R. Astron. Soc. 425, L51-L55 (2012). 1206.1861

38. Wolcott-Green, J., Haiman, Z. \& Bryan, G. L. Beyond $J_{\text {crit }}$ : a critical curve for suppression of $\mathrm{H}_{2}$-cooling in protogalaxies. Mon. Not. R. Astron. Soc., submitted (2016). 1609.02142

39. Dijkstra, M., Ferrara, A. \& Mesinger, A. Feedback-regulated supermassive black hole seed formation. Mon. Not. R. Astron. Soc. 442, 20362047 (2014). 1405.6743

40. Machacek, M. E., Bryan, G. L. \& Abel, T. Simulations of Pregalactic Structure Formation with Radiative Feedback. Astrophys. J. 548, 509521 (2001). astro-ph/0007198

41. O'Shea, B. W. \& Norman, M. L. Population III Star Formation in a $\Lambda$ CDM Universe. II. Effects of a Photodissociating Background. Astrophys. J. 673, 14-33 (2008). 0706.4416

42. Abel, T. \& Wandelt, B. D. Adaptive ray tracing for radiative transfer around point sources. Mon. Not. R. Astron. Soc. 330, L53-L56 (2002). astro-ph/0111033 
43. Górski, K. M. et al. HEALPix: A Framework for High-Resolution Dis cretization and Fast Analysis of Data Distributed on the Sphere. Astrophys. J. 622, 759-771 (2005). astro-ph/0409513

44. Wolcott-Green, J., Haiman, Z. \& Bryan, G. L. Photodissociation of $\mathrm{H}_{2}$ in protogalaxies: modelling self-shielding in three-dimensional simulations. Mon. Not. R. Astron. Soc. 418, 838-852 (2011). 1106.3523

45. Chen, P., Wise, J. H., Norman, M. L., Xu, H. \& O'Shea, B. W. Scaling Relations for Galaxies Prior to Reionization. Astrophys. J. 795, 144 (2014). 1408.2523

46. Bruzual, G. \& Charlot, S. Stellar population synthesis at the resolution of 2003. Mon. Not. R. Astron. Soc. 344, 1000-1028 (2003). astro-ph/ 0309134

47. Schaerer, D. On the properties of massive Population III stars and metal-free stellar populations. Astron. Astrophys. 382, 28-42 (2002) astro-ph/0110697

48. Abel, T., Anninos, P., Zhang, Y. \& Norman, M. L. Modeling primordial gas in numerical cosmology. New Astronomy 2, 181-207 (1997).

49. Gunn, J. E. \& Gott, J. R., III. On the Infall of Matter Into Clusters of Galaxies and Some Effects on Their Evolution. Astrophys. J. 176, 1 (1972).

50. Gisler, G. R. The fate of gas in elliptical galaxies and the density evolution of radio sources. Astron. Astrophys. 51, 137-150 (1976).

51. McCarthy, I. G. et al. Ram pressure stripping the hot gaseous haloes of galaxies in groups and clusters. Mon. Not. R. Astron. Soc. 383, 593-605 (2008). 0710.0964

52. Hosokawa, T., Omukai, K. \& Yorke, H. W. Rapidly Accreting Supergiant Protostars: Embryos of Supermassive Black Holes? ArXiv e-prints (2012). 1203.2613

53. Hosokawa, T., Yorke, H. W., Inayoshi, K., Omukai, K. \& Yoshida, N. Formation of Primordial Supermassive Stars by Rapid Mass Accretion. As trophys. J. 778, 178 (2013). 1308.4457

54. Schleicher, D. R. G., Palla, F., Ferrara, A., Galli, D. \& Latif, M. Massive black hole factories: Supermassive and quasi-star formation in primordial halos. Astron. Astrophys. 558, A59 (2013). 1305.5923

55. Fiacconi, D. \& Rossi, E. M. Bright vigorous winds as signposts of supermassive black hole birth. Mon. Not. R. Astron. Soc. 455, 2-16 (2016) 1509.07511

56. Nakauchi, D., Hosokawa, T., Omukai, K., Saio, H. \& Nomoto, K. Do Stellar Winds Prevent the Formation of Supermassive Stars by Accretion? ArXiv e-prints (2016). 1611.09601

57. Regan, J. A., Johansson, P. H. \& Haehnelt, M. G. Numerical resolution effects on simulations of massive black hole seeds. Mon. Not. R. Astron. Soc. 439, 1160-1175 (2014). 1312.4962

58. Inayoshi, K. \& Haiman, Z. Does disc fragmentation prevent the formation of supermassive stars in protogalaxies? Mon. Not. R. Astron. Soc. 445 1549-1557 (2014). 1406.5058

59. Regan, J. A. \& Haehnelt, M. G. Pathways to massive black holes and compact star clusters in pre-galactic dark matter haloes with virial tem peratures 10000K. Mon. Not. R. Astron. Soc. 396, 343-353 (2009) 0810.2802

60. Fialkov, A., Barkana, R., Visbal, E., Tseliakhovich, D. \& Hirata, C. M. The 21-cm signature of the first stars during the Lyman-Werner feedback era Mon. Not. R. Astron. Soc. 432, 2909-2916 (2013). 1212.0513

61. Visbal, E., Haiman, Z., Terrazas, B., Bryan, G. L. \& Barkana, R. Highredshift star formation in a time-dependent Lyman-Werner background Mon. Not. R. Astron. Soc. 445, 107-114 (2014). 1402.0882

62. Visbal, E., Haiman, Z. \& Bryan, G. L. Limits on Population III star formation in minihaloes implied by Planck. Mon. Not. R. Astron. Soc. 453 4456-4466 (2015). 1505.06359

63. Tseliakhovich, D. \& Hirata, C. Relative velocity of dark matter and baryonic fluids and the formation of the first structures. Phys. Rev. D 82 083520 (2010). 1005.2416

64. Stacy, A., Bromm, V. \& Loeb, A. Effect of Streaming Motion of Baryons Relative to Dark Matter on the Formation of the First Stars. Astrophys. J. Lett. 730, L1 (2011). 1011.4512

65. Fialkov, A., Barkana, R., Tseliakhovich, D. \& Hirata, C. M. Impact of the relative motion between the dark matter and baryons on the first stars: semi-analytical modelling. Mon. Not. R. Astron. Soc. 424, 1335-1345 (2012). 1110.2111

66. Naoz, S., Yoshida, N. \& Gnedin, N. Y. Simulations of Early Baryonic Structure Formation with Stream Velocity. II. The Gas Fraction. Astro phys. J. 763, 27 (2013). 1207.5515

67. Tanaka, T. L. \& Li, M. The formation of massive black holes in z 30 dark matter haloes with large baryonic streaming velocities. Mon. Not. $R$. Astron. Soc. 439, 1092-1100 (2014). 1310.0859

68. Fernandez, R., Bryan, G. L., Haiman, Z. \& Li, M. $\mathrm{H}_{2}$ suppression with shocking inflows: testing a pathway for supermassive black hole formation. Mon. Not. R. Astron. Soc. 439, 3798-3807 (2014). 1401.5803
69. Visbal, E., Haiman, Z. \& Bryan, G. L. A no-go theorem for direct collapse black holes without a strong ultraviolet background. Mon. Not. R. Astron. Soc. 442, L100-L104 (2014). 1403.1293

70. Turk, M. J. et al. yt: A Multi-code Analysis Toolkit for Astrophysical Simulation Data. Astrophys. J. Suppl. 192, 9 (2011). 1011.3514

71. Hunter, J. D. Matplotlib: A 2 d graphics environment. Computing In Sci ence \& Engineering 9, 90-95 (2007).

72. Wise, J. H. \& Cen, R. Ionizing Photon Escape Fractions From HighRedshift Dwarf Galaxies. Astrophys. J. 693, 984-999 (2009). 0808. 2477

Materials \& Correspondence Correspondence and requests for materials should be addressed to John Regan. (email: john.regan@dcu.ie).

Acknowledgements This work was supported by the Science and Technology Facilities Council (grant numbers ST/L00075X/1 and RF040365) and by NASA grant NNX15AB19G (to ZH). JR acknowledges support from the EU commission via the Marie Skłodowska-Curie Grant - "SMARTSTARS" - grant number 699941. JW is supported by National Science Foundation grants AST-1333360 and AST-1614333 and Hubble theory grants HSTAR-13895 and HST-AR-14326. PHJ acknowledges the support of the Academy of Finland grant 1274931. This work used the DiRAC Data Centric system at Durham University, operated by the Institute for Computational Cosmology on behalf of the STFC DiRAC HPC Facility (www.dirac.ac.uk). This equipment was funded by BIS National E-infrastructure capital grant ST/K00042X/1, STFC capital grant ST/H008519/1, and STFC DiRAC Operations grant ST/K003267/1 and Durham University. DiRAC is part of the $\mathrm{Na}$ tional E-Infrastructure. Some of the preliminary numerical simulations were also performed on facilities hosted by the CSC -IT Center for Science in Espoo, Finland, which are financed by the Finnish ministry of education. The Center for Computational Astrophysics is supported by the Simons Foundation. The freely available astrophysical analysis code y: ${ }^{70}$ and plotting library matplotlit $\sqrt{71}$ was used to construct numerous plots within this paper.

Author contributions JR modified the publicly available Enzo code and Grackle codes used in this work, ran and analysed the code results, and wrote the initial manuscript. JR, ZH, JW \& EV determined the simulation setup. The radiation particle model was conceived and designed by JR, PHJ \& JW. All authors contributed to the interpretation of the results, and to the text of the final manuscript.

Competing Interests The authors declare that they have no competing financial interests. 


\section{Supplementary Information}

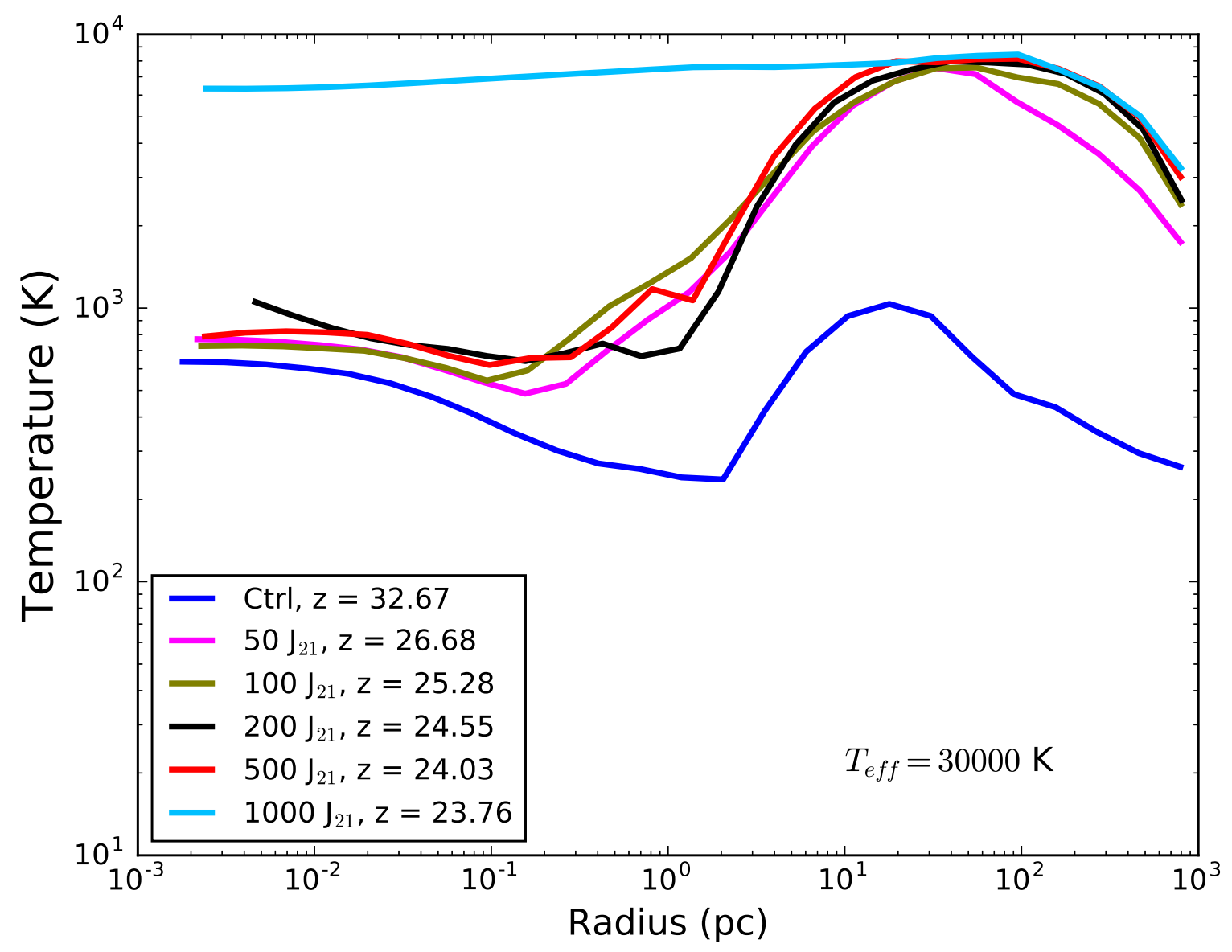

Figure 1 Supplementary Data: Background Radiation Fields. Radial profile of the temperature for different background field strengths. The background field is modelled as a blackbody with an effective temperature of $30000 \mathrm{~K}$. The bright nearby source is not included in these simulations, and the radiation is purely that of the longer-lived background radiation field. The "Ctrl" field represents the no-background case, with the background field strength then increasing up to $1000 J_{21}$. For this halo realisation, the critical field strength is reached when the background field is $\sim 1000 J_{21}$. Achieving such a high background field in the high-z Universe is not viable, and in practice what will be required is a background augmented by an excess of local source(s), by unusually large streaming motions between baryons and dark matter, and/or by an unusually rapid merger history (see main text for discussion). 
Table 1 | Supplementary Data Table: Simulation Details

\begin{tabular}{|l|c|l|l|l|l|l|l|}
\hline \hline Sim Name $^{\mathrm{a}}$ & Background $^{\mathrm{b}}$ & $\mathrm{z}_{\text {on }}{ }^{\mathrm{c}}$ & $z_{\text {collapse }}^{d}$ & $T_{\text {sync }}^{e}$ & $T_{\text {on }}^{f}$ & $\mathrm{R}_{\text {sep }}^{\mathrm{g}}$ & Result $^{\mathrm{h}}$ \\
\hline z2550_100_350 & 100 & 25.50 & 24.37 & 1.59 & 8.53 & 322 & Molecular \\
z2550_100_250 & 100 & 25.50 & 24.19 & 1.59 & 9.97 & 251 & Atomic \\
z2540_100_350 & 100 & 25.40 & 24.40 & 0.87 & 7.51 & 322 & Molecular \\
z2540_100_250 & 100 & 25.40 & 24.20 & 0.87 & 9.13 & 276 & Atomic \\
z2530_100_350 & 100 & 25.30 & 24.45 & 0.14 & 6.50 & 321 & Molecular \\
z2530_100_250 & 100 & 25.30 & 24.25 & 0.14 & 8.05 & 242 & Molecular \\
z25285_100_350 & 100 & 25.285 & 24.45 & 0.04 & 6.39 & 321 & Atomic \\
z25285_100_250 & 100 & 25.285 & 24.26 & 0.04 & 7,91 & 242 & Molecular \\
\hline z2530_150_300 & 150 & 25.30 & 24.06 & 3.37 & 9.66 & 303 & Atomic \\
z2515_150_200 & 150 & 25.15 & 23.98 & 2.27 & 9.30 & 218 & Atomic \\
z2500_150_350 & 150 & 25.00 & 24.38 & 1.14 & 4.78 & 388 & Molecular \\
z2500_150_250 & 150 & 25.00 & 24.10 & 1.14 & 7.10 & 237 & Atomic \\
z2490_150_350 & 150 & 24.90 & 24.41 & 0.38 & 3.83 & 388 & Atomic \\
z2490_150_250 & 150 & 24.90 & 24.18 & 0.38 & 5.68 & 236 & Atomic \\
z2486_150_350 & 150 & 24.86 & 24.46 & 0.1 & 3.12 & 388 & Atomic \\
z2486_150_250 & 150 & 24.86 & 24.23 & 0.07 & 4.88 & 235 & Atomic \\
\hline z2500_200_250 & 200 & 24.62 & 24.15 & 3.47 & 6.69 & 390 & Molecular \\
z2500_200_350 & 200 & 24.62 & 23.93 & 3.47 & 8.51 & 238 & Atomic \\
z2480_200_300 & 200 & 24.65 & 24.24 & 1.95 & 4.43 & 388 & Molecular \\
z2480_200_400 & 200 & 24.65 & 23.99 & 1.95 & 6.48 & 262 & Atomic \\
z2460_200_250 & 200 & 24.60 & 24.33 & 0.40 & 2.15 & 387 & Molecular \\
z2460_200_400 & 200 & 24.60 & 24.16 & 0.40 & 3.53 & 235 & Atomic \\
z2457_200_250 & 200 & 24.57 & 24.15 & 0.16 & 3.37 & 360 & Atomic \\
z2457_200_400 & 200 & 24.57 & 23.93 & 0.16 & 5.20 & 262 & Atomic \\
z24555_200_250 & 200 & 24.555 & 24.39 & 0.007 & 1.31 & 387 & Molecular \\
z24555_200_400 & 200 & 24.555 & 24.26 & 0.007 & 2.36 & 309 & Atomic \\
\hline
\end{tabular}

The above table contains the simulation name ${ }^{a}$, the uniform background radiation field in units of $\mathbf{J}_{21}^{b}$, the redshift at which the primary halo turns "on"c , the collapse redshift of the secondary halo ${ }^{d}$, the synchronisation time in Myrs ${ }^{e}$, the time for which the primary halo was on in $\mathrm{Myrs}^{f}$, the average separation, in parsecs, between the primary and the secondary ${ }^{g}$ and finally the result of the collapse (i.e. an atomic collapse or a molecular collapse ${ }^{h}$ ). All units are physical, unless explicitly stated otherwise.

Table 2 | Supplementary Data Table: Radiation Spectrum

\begin{tabular}{|l|l|l|l|}
\hline \hline Source & L [ph/s] & Energy Bins [Ev] & Energy Fraction \\
\hline Metal Enriched & $1.2 \times 10^{52}$ & $2.0,12.8,14.55,25.05$ & $0.7510,0.1080,1.33 \times 10^{-7}, 2.54 \times 10^{-3}$ \\
PopIII & $1.0 \times 10^{52}$ & $2.0,12.8,18.37,47.23$ & $0.4585,0.1509,2.88 \times 10^{-4}, 4.77 \times 10^{-2}$ \\
\hline
\end{tabular}

The radiation spectra for both the metal enriched galaxy model we used in this study as well as a comparable PopIII model. The spectra are convolved with a shielding function which is based on a simple model of interstellarmedium extinction and accounts for absorption of the ionising radiation within the primary halo itself. The density distribution and emission spectra are based on the high resolution simulations of Wise \& Cen (2009) ${ }^{72}$. The model convolves the spectral energies (above $13.6 \mathrm{eV}$ ) with a simple modelling of the optical depth to ionising radiation ${ }^{21}$. 